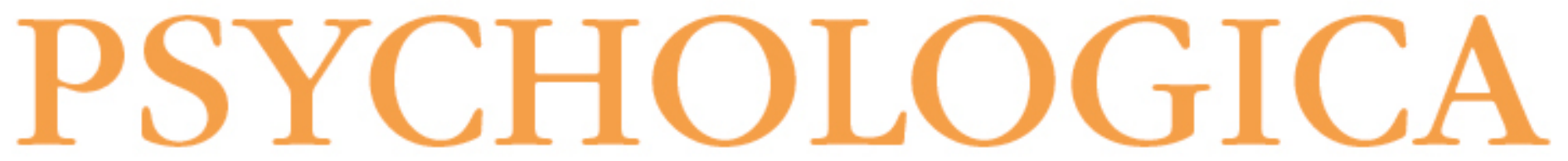

Relações entre desempenho escolar e interesses profissionais de estudantes do ensino médio: desempenho escolar e interesses profissionais

Autor(es): $\quad$ Noronha, Ana Paula Porto; Ambiel, Rodolfo Augusto Matteo

Publicado por: Imprensa da Universidade de Coimbra

URL

persistente: URI:http://hdl.handle.net/10316.2/5418

DOI: $\quad$ DOI:http://dx.doi.org/10.14195/1647-8606_50_14

Accessed : $\quad$ 26-Apr-2023 11:37:52

A navegação consulta e descarregamento dos títulos inseridos nas Bibliotecas Digitais UC Digitalis, UC Pombalina e UC Impactum, pressupõem a aceitação plena e sem reservas dos Termos e Condições de Uso destas Bibliotecas Digitais, disponíveis em https://digitalis.uc.pt/pt-pt/termos.

Conforme exposto nos referidos Termos e Condições de Uso, o descarregamento de títulos de acesso restrito requer uma licença válida de autorização devendo o utilizador aceder ao(s) documento(s) a partir de um endereço de IP da instituição detentora da supramencionada licença.

Ao utilizador é apenas permitido o descarregamento para uso pessoal, pelo que o emprego do(s) título(s) descarregado(s) para outro fim, designadamente comercial, carece de autorização do respetivo autor ou editor da obra.

Na medida em que todas as obras da UC Digitalis se encontram protegidas pelo Código do Direito de Autor e Direitos Conexos e demais legislação aplicável, toda a cópia, parcial ou total, deste documento, nos casos em que é legalmente admitida, deverá conter ou fazer-se acompanhar por este aviso. 


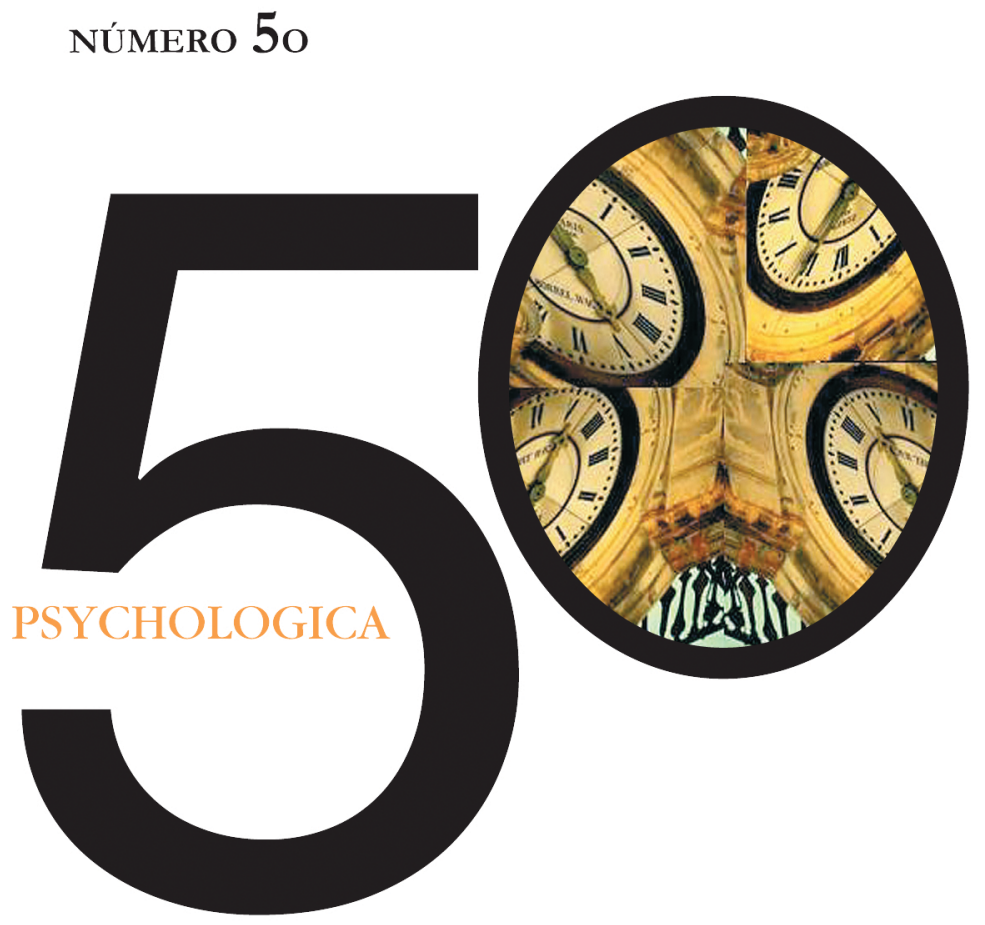

IMPRENSA DA UNIVERSIDADE DE COIMBRA

FACULDADE DE PSICOLOGIA E DE CIÊNCIAS DA EDUCAÇÃO DA UNIVERSIDADE DE COIMBRA 


\title{
Relações entre desempenho escolar e interesses profissionais de estudantes do ensino médio Desempenho escolar e interesses profissionais
}

\author{
Ana Paula Porto Noronha' \& Rodolfo Augusto Matteo Ambiel ${ }^{2}$
}

\begin{abstract}
Esse artigo buscou estudar as relações entre interesses profissionais e notas de estudantes do ensino médio de uma escola particular do estado de Minas Gerais e as diferenças de médias entre gêneros. Para tanto, 245 estudantes (24,1\% da primeira série, $40,8 \%$ da segunda, e $35,1 \%$ da terceira) participaram da pesquisa, com idade média de 16,3 anos ( $D P=0,9$ ), sendo que $60,8 \%$ eram mulheres. Foram utilizadas a Escala de Aconselhamento Profissional (EAP) e as notas das avaliações dos dois primeiros bimestres letivos de 2005. Os resultados indicaram correlações significativas coerentes, com relações positivas entre disciplinas e áreas de interesse afins, e correlações negativas entre áreas com poucas relações de conteúdo. Observou-se um melhor desempenho escolar das mulheres e uma tendência de preferência por atividades típicas de cada gênero. Implicações práticas dos achados e propostas de novos estudos no sentido de clarificar tais relações são indicadas.
\end{abstract}

PALAVRAS-CHAVE: Ensino Médio; Diferenças de Gênero; Avaliação Psicológica, Orientação Profissional.

\section{Introdução}

Historicamente, o campo da Orientação Profissional (OP) esteve circunscrito, de alguma forma, às instituições educacionais, profissionalizantes ou não. Exemplo disso pode ser encontrado nas palavras de Santos (1997), que relata a iniciativa do Liceu de Artes e Ofícios de São Paulo, em 1924, no sentido da criação de uma seção para orientação e seleção, voltada às pessoas que se interessavam por seus cursos. No mesmo sentido, a Estrada de Ferro Sorocabana, em 1930, e o Serviço Nacional de Aprendizgem Industrial (SENAI), na década de 1940, também fundaram

\footnotetext{
1 Doutora em Psicologia Ciência e Profissão pela Pontifícia Universidade Católica de Campinas. Docente do Programa de Pós-Graduação Stricto Sensu em Psicologia da Universidade São Fraancisco. Bolsista Produtividade em Pesquisa do CNPq. (ana.noronha@saofrancisco.edu.br)

2 Bolsista de Iniciação Científica PIBIC/CNPq; acadêmico do $9 .{ }^{\circ}$ semestre do Curso de Psicologia da Universidade São Francisco. (ambielram@yahoo.com.br)
} 
institutos especializados em seleção, orientação e educação profissional de seus funcionários (Noronha, Sisto \& Santos, 2007; Abade, 2005).

O final da década de 1940 foi marcado pela inauguração do Instituto de Seleção e Orientação Profissional (ISOP) no Rio de Janeiro, por iniciativa da Fundação 278 Getúlio Vargas. A finalidade do ISOP era a de ajustar os trabalhadores às funções disponíveis no mercado (Abade, 2005), utilizando técnicas científicas para tal tarefa, o que fez do instituto um importante centro construtor de testes psicológicos e de desenvolvimento de técnicas para seleção de pessoal (Noronha, Sisto, \& Santos, 2007). Também nesta época, a criação do ISOP no Rio de Janeiro, acabou por influenciar a criação do Serviço de Orientação e Seleção Profissional (SOSP), em Belo Horizonte. As propostas de ambas as iniciativas eram semelhantes, tendo um foco psicométrico na avaliação e seleção profissional.

É importante resgatar que tão logo a profissão de psicólogo no Brasil foi oficializada, em 1962, diversos cursos foram formados, sendo que muitos deles contavam com disciplinas de orientação e seleção profissional em seus currículos, o que auxiliou no desenvolvimento da área de OP (Freitas, 1973). Segundo Abade (2005), nessa época a atuação já não era ligada exclusivamente às técnicas psicométricas, adquirindo também um caráter clínico, com a influência principalmente das propostas de Carl Rogers, nos Estados Unidos, e da Psicanálise, na Europa.

Entretanto, a ascenção da ditadura militar no Brasil, da metade para o final da década de 1960, fez com que muitos dos avanços técnicos da psicologia, que vinham se estabelecendo até então estagnassem, e a falta de interesse e investimento em educação e cultura fez com que tal estancamento fosse sentido em todos os setores da sociedade. Apenas na década de 1980, houve movimentos no sentido de uma reerguida cultural do país, o que também refletiu na psicologia, e mais especificamente na OP (Noronha, Sisto, \& Santos, 2007).

Essa afirmação também pode ser constatada no trabalho de Melo-Silva (1999), que identificou que a retomada da área, no tocante às publicações, iniciou-se na referida década, tendo se consolidado na década de 1990. A autora frisa que as publicações do tipo relatos de experiência sobre intervenções em OP foram predominantes nesse período. Na mesma direção, Abade (2005), encontrou dados mostrando ainda que há diversidade quanto à abordagem adotada pelos profissionais, sendo as mais comuns, a psicométrica e a clínica.

O trabalho de Noronha e Ambiel (2006) buscou avaliar a produção científica sobre orientação profissional disponível em bases de dados eletrônicas do Brasil, entre 1950 e 2005. Além de confirmar os resultados de Melo-Silva (1999) e Abade (2003) quanto ao crescimento da área, o estudo mostrou que houve evoluções no número de publicações teóricas e de pesquisa ao longo das décadas, bem 
como uma preocupação evidente de se avaliar processos, técnicas e instrumentos utilizados em OP.

Outra análise importante encontrada no estudo foi quanto aos construtos psicológicos mais investigados nos artigos científicos no decorrer dos 55 anos ponderados pelos autores. Entre os testes utilizados, os de interesse foram predominantes, seguidos, em ordem decrescente, pelos de personalidade, maturidade e inteligência, sendo que até 2005, apenas um instrumento de avaliação de interesses constava na lista de testes aprovados pelo Conselho Federal de Psicologia (Noronha \& Ambiel, 2006).

Apesar dessa predominância, a situação dos testes de interesse disponíveis para comercialização e uso no Brasil antes das Resoluções do CFP (2001; 2003) era preocupante. Tais resoluções afirmam que somente instrumentos com, no mínimo, uma evidência de validade e outra de precisão, além de estudos de padronização, poderiam ser vendidos e usados profissionalmente. Ottati, Noronha e Salviatti (2003) analisaram as informações a respeito da validade, precisão e padronização contidas nos manuais de sete instrumentos de avaliação de interesses profissionais, encontrando dados alarmantes. Com relação à validade, apenas dois instrumentos traziam as informações, e de forma precária. Sobre precisão, apenas um instrumento mostrava os estudos feitos, e no que toca à padronização, apenas três dos sete instrumentos avaliados faziam menção a esses estudos.

Buscando contribuir com a área, Noronha, Sisto e Santos (2007) construíram a Escala de Aconselhamento Profissional (EAP), composta de 61 itens, em formato likert de cinco pontos, sendo que contêm em seu teor atividades profissionais diversas, típicas de mais de uma profissão. No processo de construção, os autores partiram do princípio de que as pessoas podem gostar de realizar atividades além daquelas típicas de sua ocupação ou profissão. A finalidade do instrumento é fornecer um perfil profissional, caracterizando níveis e intensidades de preferência por determinados grupos de atividades. Dessa forma, os autores assumiram que os interesses profissionais podem ser compreendidos como preferência por algumas atividades laborais, encontrando respaldo nas afirmações de Anastasi e Urbina (2000) e, de Savickas (1999), cujas asserções são apresentadas a seguir.

O último autor informa que o termo interesse tem origem no latim, e significa "estar entre", denotando uma relação de atração entre o indivíduo e algo do ambiente, de forma que aquilo que atrai, o faz por parecer vantajoso ou benéfico. Assim, os interesses assumiriam um papel motivacional de intermediação entre o sujeito e seu meio.

Savickas (1999) faz ainda referências às teorias da aprendizagem, que afirmam que o indivíduo tende a preferir, ou seja, é atraído por atividades com as quais 
teve experiências gratificantes, julgando ser habilidoso nesse domínio e esperando resultados favoráveis de sua execução. Em adição, citando Gottfredson (1981), o autor afirma que esse processo de aprendizagem parece ser intermediado por fatores como sexo, raça e classe social. Dessa forma, Savickas (1999) considera que os inventários de interesse, em geral, definem o construto como padrões de preferência, oposição ou indiferença a determinados estímulos ambientais.

Atualmente, o Conselho Federal de Psicologia (CFP, 2008) indica que poucos instrumentos de avaliação no contexto vocacional estão aptos para ser usados profissionalmente. Um deles, cuja descrição detalhada encontra-se a seguir, é a Escala de Aconselhamento Profissional (EAP, Noronha, Sisto e Santos, 2007), utilizada nesse estudo.

Buscando avaliar as propriedades psicométricas do EAP, os autores realizaram estudos de validade e precisão, cuja amostra foi composta por 762 estudantes (59\% mulheres; idade média de 24,1 anos), de 13 diferentes cursos universitários de Minas Gerais e São Paulo. Com relação às evidências de validade, os autores desenvolveram estudos de estrutura interna e de critério.

Para a obtenção das evidências relativas à estrutura interna dos itens, realizou-se uma análise fatorial, que indicou a existência de sete dimensões, denominadas Ciências Exatas (14 itens), Artes e Comunicação (14 itens), Ciências Biológicas e da Saúde (9 itens), Ciências Agrárias e Ambientais (13 itens), Atividades Burocráticas (13 itens), Ciências Humanas e Sociais Aplicadas (10 itens) e Entretenimento (seis itens), cujos índices de saturação foram superiores a 0,30 e variância explicada de $57,31 \%$. Devido à natureza dos itens, muitos deles apresentaram cargas fatoriais aceitáveis em mais de um fator.

No tocante à validade de critério, os autores compararam as médias obtidas pelos participantes em cada dimensão em relação aos cursos deles, visando observar quais carreiras se destacam em cada dimensão. Em Ciências Exatas, observou-se uma prevalência das médias das Engenharias e Administração, e menores médias de fisioterapia e educação artística. Na dimensão Artes e Comunicação, Educação Artísitica e Jornalismo obtiveram as maiores médias, enquanto Medicina e Veterinária encontraram-se no outro extremo. Em Ciências Biológicas e da Saúde, Medicina e Fisioterapia se destacaram, em oposição à Educação Artística, Jornalismo e Engenharias.

Na quarta dimensão, Ciências Agrárias e Ambientais, Veterinária e Turismo tiveram médias mais altas, enquanto que Educação Artística e Jornalismo tiveram as mais baixas. Na dimensão Atividades Burocráticas, Administração se sobressaiu, ao lado disso, Educação Artística e Medicina obtiveram médias baixas. Em Ciências Humanas e Sociais Aplicadas, a predominância foi de Pedagogia, Jornalismo e 
Psicologia, enquanto que Veterinárias e Engenharias não se destacaram. Por fim, a sétima dimensão, Entretenimento, mostrou que Turismo obteve a maior média, enquanto que para Medicina, as médias se mostraram baixas.

No manual do instrumento, os autores concluíram que o instrumento possui evidências satisfatórias de validade. Pôde-se perceber que em maior ou menor intensidade, os sujeitos demonstraram preferir atividades de diversas áreas, embora se possa dizer que houve uma dominância de determinadas carreiras em dimensões que continham atividades típicas delas.

No que tange à precisão, quatro procedimentos foram utilizados para estimá-la. Segundo o modelo de Rasch, as dimensões apresentaram variação entre 0,97 e 0,99 para os itens entre 0,76 e o,88 para as pessoas, indices considerados altos. Os indives de alfa de Cronbach variaram entre 0,79 e 0,94, enquanto os de Spearman-Brown e de Guttman ficaram entre 0,75 e 0,91. Por tais resultados, pode-se considerar que a EAP é uma escala, além de válida, também precisa.

Ainda em relação às pesquisas com o instrumento, Sartori (2007) aplicou em 132 estudantes de ensino médio, sendo 50\% de cada gênero, e com média de idade de 15,9 anos. Quanto à distribuição por série, $47 \%$ cursavam o primeiro ano do ensino médio, $36,4 \%$ o segundo e $15,9 \%$ o terceiro ano. O objetivo do trabalho foi correlacionar o instrumento com o Self-Directed Search (SDS, Holland, Powell \& Fritzsche, 1994), que também avalia interesses profissionais por meio dos perfis tipológicos, segundo a teoria de Holland (1963), além de analisar a variância dos escores no EAP, por série.

As correlações entre os instrumentos revelaram significâncias, dentre elas, a dimensão Ciências Exatas com os tipos Realista e Investigativo. Já a dimensão Artes e Comunicação correlacionou-se com os tipos Artístico e Social, também de forma positiva. A terceira dimensão, Ciências Biológicas e da Saúde, obteve indices de correlação positivos com Investigativo e Social, da mesma forma que a dimensão quatro, Ciências Agrárias e Ambientais, que se correlacionou com os mesmos tipos.

A dimensão Atividades Burocrática mostrou-se correlacionada principalmente com Convencional e Empreendedor. Já a quinta dimensão, Ciências Humanas e Sociais Aplicadas, correlacionou-se com Investigativo, Artístico e Social, enquanto que a última dimensão, Entretenimento, correlacionou-se com Social, Artístico e Empreededor. No que diz respeito à variância das médias do EAP tanto em relação às séries quanto aos sexos, cabe destacar que não foram encontradas diferenças significativas. Para a autora, tal achado sugere que a escolaridade e o gênero não pareceram relacionar-se diretamente com o nível de interesse dos sujeitos dessa amostra. 
Dentre outras pesquisas relevantes, destaca-se a de Fogliatto, Pérez, Olaz e Parodi (2003). Os autores pesquisaram as propriedades psicométricas do Cuestionario de Interesses Profesionales Revisado (CIP-R). O instrumento, que tem o mesmo princípio teórico do EAP, avalia os interesses por meio de 114 itens distruídos em 15 escalas (Cálculo, Científica, Desenho, Tecnológica, Geoastronômica, Naturalista, Sanitária, Assistencial, Jurídica, Econômica, Comunicacional, Humanística, Artística, Musical e Lingüística). A estabilidade temporal da escala foi avaliada, sendo que 156 estudantes secundários (68,8\% mulheres; idade média de 17,50$)$ responderam ao instrumento no início e no final do ano letivo, e os coeficientes das escalas variaram entre 0,78 e 0,90 , considerados adequados. A consistência interna também foi avaliada, e os alfas variaram entre 0,83 e 0,91, indicando que a escala apresenta boa precisão.

Com relação à validade, a estrutura interna foi avaliada a partir das respostas de 810 estudantes, chegando-se à solução de 15 fatores. Ainda, os autores compararam os escores de homens e mulheres, buscando avaliar as médias de grupos contrastantes. A amostra feminina pontuou mais nas escalas Comunicacional, Jurídica, Humanística, Assistencial, Sanitária e Artística, enquanto que os homens se destacaram nas escalas Tecnológica, Naturalista, Cálculo, Geoastronômica, Ciências Básicas e Desenho. Indicando essa análise como verificação da validade, os autores concluíram que homens pontuaram mais em escalas relativas a profissões tipicamente masculinas, enquanto que as mulheres tiveram médias mais altas em atividades com tendências femininas. Por fim, os autores procederam a entrevista telefônica com metade da amostra que respondeu ao instrumento, seis meses depois da aplicação, buscando investigar a congruência entre os interesses avaliados e a eleição de carreira. Os resultados indicaram correlações positivas entre a eleição de carreira e os interesses avaliados, evidenciando que a escala apresenta bom poder preditivo.

Com o intuito de mensurar as diferenças de gênero na preferência e atuação profissional, alguns estudos foram realizados, por diferentes meios. Por exemplo, Welter (2007) aplicou o Teste de Fotos de Profissões (BBT) em 204 pessoas (59,8\% homens), em situação de seleção ou orientação profissional, dos quais 143 eram adultos. Diferenças significativas entre as faixas etárias foram identificadas, sendo que parece haver uma tendência para o aumento de escolhas positivas das fotos de profissões com o passar dos anos. Além disso, a análise da estrutura de inclinação por gênero indicou que as mulheres parecem preferir profissões caracterizadas por cuidados com os outros, relações sociais amplas e afetivas, trabalho em equipe e com pouca tendência à rotina, objetividade, racionalidade e força física. Já os homens pareceram estar mais propensos a preferir profissões em que possam se expressar de forma dinâmica e corajosa, de forma lógica e com senso de realidade, com criatividade e auto-exposição. 
Ainda com relação às variáveis relacionadas ao processo de escolha profissional, Neiva, Silva, Miranda e Esteves (2005) observaram as diferenças de nível de maturidade para escolha profissional em estudantes de ensino médio. Os sujeitos foram 950 alunos (51\% homens; média de 16,7 anos) de escolas particular $(60,9 \%)$ e pública de Curitiba. A avaliação foi feita por meio da Escala de Maturidade para Escolha Profissional (EMEP), e revelou que parece existir diferenças significativa entre sexo, sendo que as mulheres apresentam médias mais altas, especialmente nas subescalas Responsabilidade e Autoconhecimento. Além disso, estudantes de escola particular também apresentaram médias maiores que os de escola pública em todas as subescalas, bem como alunos da segunda série em relação à primeira, e da terceira em relação à segunda, mas apenas nas subescalas Determinação e Independência.

Com relação ao desempenho acadêmico, avaliações desse tipo têm sido feitas em países da América do Sul. No tocante à relação entre depressão e desempenho em línguas e matemática de adolescentes argentinos, Pérez e Urquijo (2001) indicaram que tal relação depende de interações com idade, sexo e tipo de escola, parecendo afetar principalmente mulheres e alunos de escolas públicas. $\mathrm{Na}$ Colômbia, Plazas, Penso e López (2006) buscaram avaliar se status sociométrico, avaliado por meio da denominação dada por pares, e gênero exerciam algum efeito sobre o rendimento acadêmico de 156 estudantes (88 mulheres) de um colégio público. Os resultados indicaram que variáveis como impacto e preferência sociais influenciam significativamente os rendimento escolar, embora o faça de forma diferenciada para homens e mulheres.

O estudo de Dias, Enumo e Turini (2006) buscou avaliar habilidades de escrita, leitura e aritmética de 170 estudantes (52,9\% mulheres, com idades entre oito e 19 anos) de segunda à quinta série de uma escola pública de Vitória (ES), por meio do Teste de Desempenho Escolar. Foram feitas duas avaliações com os mesmos alunos, num intervalo de um ano. De forma geral, pôde-se perceber que os alunos tiveram uma melhora significativa quando comparadas as duas avaliações. Ainda, em todas as medidas as meninas tiveram desempenho melhor, com médias significativamente diferentes e maiores em relação aos meninos.

Tendo em vista o exposto, parece claro que as investigações a respeito dos interesses profissionais têm sido bastante exploradas. No entanto, o objeto de investigação do presente trabalho versa sobre as relações entre os interesses e o desempenho acadêmico de estudantes de ensino médio, clientela característica de programas de OP desenvolvidos no Brasil, tal como proposto por Melo-Silva, Bomfim, Esbrogeo e Soares, 2003 e Neiva, Silva, Miranda e Esteve, 2006. Mais especialmente, essa pesquisa visa estudar as relações entre as notas em disciplinas curriculares das três séries do ensino médio com as preferências profissionais, avaliadas pelo EAP, além das diferenças entre gêneros. 


\section{Método}

\section{Participantes}

Participaram da presente pesquisa 245 estudantes de ensino médio de uma escola particular do interior de Minas Gerais. As idades, na amostra total, variaram entre 14 e 19 anos, sendo a média igual a 16,3 anos ( $D P=0,9)$. Quanto ao sexo, 6o,8\% dos participantes eram mulheres. Os estudantes eram oriundos das três séries, e divididos da forma apresentada na Tabela 1.

Tabela 1. Caracterização da amostra, por série

\begin{tabular}{ccccccccc}
\hline Série & \multicolumn{2}{c}{ Amostra } & \multicolumn{3}{c}{ Sexo } & \multicolumn{3}{c}{ Idade } \\
\cline { 2 - 9 } & $\mathrm{N}$ & $\%$ & $\mathrm{H}$ & $\mathrm{M}$ & Mínima & Máxima & Média & DP \\
\hline $1 .{ }^{a}$ & 59 & 24,1 & 25 & 33 & 14 & 18 & 15,3 & 0,6 \\
$2{ }^{a}$ & 100 & 40,8 & 29 & 71 & 15 & 19 & 16,3 & 0,6 \\
$3 .{ }^{a}$ & 86 & 35,1 & 41 & 45 & 16 & 19 & 17,2 & 0,6 \\
\hline
\end{tabular}

\section{Instrumentos}

Escala de Aconselhamento Profissional (EAP, Noronha, Sisto, \& Santos, 2007)

Inicialmente, o EAP contava com 220 itens gerados a partir das descrições de 65 profissões, tal como disponibilizado por guias profissionais e descrições dos perfis dos cursos de formação, disponibilizados em sites de universidades brasileiras. Tais itens foram submetidos a uma análise heurística dos itens realizada pelos autores do instrumento, sendo que permaneceram no instrumento os itens que versavam sobre atividades profissionais exercidas por mais de uma profissão, cuidando para que todas as áreas profissionais estivessem representadas. Depois dessa análise, restaram 61 itens que compuseram, por fim, a escala, que é em formato Likert, variando de freqüentemente (5) a nunca (1). Estudos de validade e precisão do EAP foram destacados na introdução do presente trabalho.

\section{Notas escolares}

As notas foram obtidas na secretaria da escola e correspondem às avaliações ocorridas no primeiro e segundo bimestres letivos. Por critérios da direção, as notas no primeiro semestre variavam entre o e 20 , enquanto que no segundo, a variação era entre o e 25 . A exceção fica por conta de Redação, que no primeiro bimestre valeu até 70 , sendo igual às demais no segundo bimestre. Os critérios valiam para as três séries. 


\section{Procedimento}

Após o projeto ter sido aprovado pelo Comitê de Ética em Pesquisa de uma instituição de ensino superior, estabeleceu-se um contato com a direção da escola, a fim de pedir autorização para a aplicação dos instrumentos, e o acesso às notas dos alunos. Uma vez recebida a autorização, mais uma visita à escola foi feita, na qual se explicou o projeto para os alunos. Esses, por sua vez, receberam duas vias do Termo de Consentimento Livre e Esclarecido, e foi solicitado a eles que levassem a um responsável, devendo devolver assinado no próximo encontro. $\mathrm{Na}$ semana seguinte, os alunos que receberam o consentimento para participar da pesquisa, responderam ao instrumento em sala de aula, sendo que cada turma levou aproximadamente 15 minutos para finalizar.

\section{Resultados}

Abaixo são oferecidos e discutidos os resultados obtidos no estudo. Inicialmente são apresentados os relativos à amostra total, incluindo correlações entre EAP e notas com todos os participantes, e por gênero. Ainda, são dadas as diferenças de médias entre gêneros, tanto com relação aos interesses quanto as notas. Em seguida, os resultados das séries separadamente também são mostrados.

Considerando os estudantes dos três anos do ensino médio (Tabela 2), observou-se que poucas correlações significativas entre as notas escolares e as dimensões do EAP foram encontradas. Dentre elas, duas são negativas, a saber, Ciências Exatas e História $(r=-0,15 ; p<0,05)$ e Entretenimento e Química $(r=-0,13 ; p<0,05)$. Ainda, três correlações positivas foram encontradas, entre a dimensão Ciências Humanas e Sociais Aplicadas com Física $(r=0,13 ; p<0,05)$, História $(r=0,18 ; p<0,01)$ e Redação $(r=0,15 ; p<0,01)$.

Ao se tomar apenas os homens das três séries, cinco correlações negativas foram encontradas. A dimensão Ciências Biológicas e da Saúde se correlacionou negativamente com Português $(r=-0,23 ; p<0,05)$ e Redação $(r=-0,24 ; p<0,05)$, enquanto que Entretenimento mostrou-se correlacionado negativamente com Matemática $(r=-0,24 ; p<0,05)$, História $(r=-0,22 ; p<0,05)$ e Inglês $(r=-0,27 ; p<0,01)$. Com relação aos escores das mulheres das três séries, Ciências Agrárias e Ambientais obteve coeficiente significativo com as notas em Química ( $r=0,19 ; p<0,05)$, enquanto que interesses em Ciências Humanas e Sociais Aplicadas correlacionaram-se com Português $(r=0,22 ; p<0,01)$, História $(r=0,20 ; p<0,05)$ e Redação $(r=0,20 ; p<0,05)$. 
Tabela 2. Correlações entre notas das três séries e dimensões da EAP

\begin{tabular}{|c|c|c|c|c|c|c|c|c|c|c|c|}
\hline Gênero & EAP & Matemática & Química & Fisica & Biologia & Português & Literatura & História & Geografia & Inglês & Redação \\
\hline \multirow[t]{7}{*}{ Geral } & CiEx & $-0,02$ & 0,05 & $-0,04$ & $-0,09$ & $-0,03$ & $-0,02$ & $-0,15^{*}$ & $-0,07$ & $-0,03$ & $-0,07$ \\
\hline & ArtCom & $-0,08$ & $-0,07$ & $-0,03$ & $-0,05$ & 0,08 & $-0,00$ & 0,06 & $-0,03$ & $-0,01$ & 0,01 \\
\hline & CiBS & 0,09 & 0,10 & 0,05 & 0,12 & $-0,03$ & 0,11 & 0,06 & 0,05 & 0,02 & $-0,00$ \\
\hline & CiAgAm & 0,07 & 0,08 & $-0,00$ & 0,02 & 0,01 & 0,06 & $-0,01$ & 0,01 & $-0,08$ & $-0,02$ \\
\hline & Bur & $-0,02$ & 0,05 & 0,05 & $-0,07$ & $-0,02$ & $-0,05$ & $-0,10$ & $-0,02$ & 0,02 & $-0,05$ \\
\hline & $\mathrm{CieHS}$ & 0,10 & 0,08 & $0,13^{*}$ & 0,00 & 0,11 & 0,08 & $0,18^{* *}$ & 0,11 & 0,08 & $0,15^{*}$ \\
\hline & Ent & $-0,13$ & $-0,13^{*}$ & $-0,08$ & $-0,074$ & 0,03 & $-0,10$ & $-0,07$ & $-0,07$ & $-0,06$ & $-0,03$ \\
\hline \multirow[t]{7}{*}{ Homens } & CiEx & $-0,04$ & 0,01 & $-0,01$ & $-0,09$ & $-0,06$ & 0,06 & $-0,14$ & $-0,15$ & 0,01 & 0,01 \\
\hline & ArtCom & $-0,20$ & $-0,18$ & $-0,16$ & $-0,13$ & $-0,08$ & $-0,11$ & $-0,04$ & $-0,14$ & $-0,20$ & $-0,08$ \\
\hline & CiBS & 0,05 & $-0,08$ & $-0,09$ & $-0,11$ & $-0,23^{*}$ & $-0,03$ & $-0,20$ & $-0,14$ & $-0,12$ & $-0,24^{*}$ \\
\hline & CiAgAm & 0,00 & $-0,09$ & $-0,09$ & $-0,16$ & $-0,14$ & $-0,06$ & $-0,17$ & $-0,14$ & $-0,22$ & $-0,19$ \\
\hline & Bur & $-0,04$ & 0,09 & 0,15 & $-0,04$ & $-0,00$ & 0,17 & $-0,06$ & $-0,07$ & 0,06 & 0,09 \\
\hline & CieHS & $-0,016$ & $-0,03$ & 0,05 & $-0,12$ & $-0,11$ & $-0,01$ & 0,06 & $-0,02$ & $-0,13$ & $-0,01$ \\
\hline & Ent & $-0,24^{*}$ & $-0,18$ & $-0,16$ & $-0,19$ & $-0,11$ & $-0,14$ & $-0,22^{*}$ & $-0,20$ & $-0,27^{* *}$ & $-0,11$ \\
\hline \multirow[t]{7}{*}{ Mulheres } & CiEx & 0,03 & 0,13 & 0,02 & 0,03 & 0,04 & 0,05 & $-0,02$ & 0,06 & 0,07 & $-0,00$ \\
\hline & ArtCom & $-0,03$ & $-0,04$ & 0,01 & $-0,06$ & 0,14 & 0,01 & 0,07 & $-0,01$ & 0,04 & 0,05 \\
\hline & CiBS & 0,07 & 0,15 & 0,06 & 0,15 & 0,01 & 0,08 & 0,08 & 0,08 & $-0,01$ & 0,09 \\
\hline & CiAgAm & 0,11 & $0,19^{*}$ & 0,05 & 0,15 & 0,11 & 0,13 & 0,09 & 0,13 & 0,00 & 0,11 \\
\hline & Bur & 0,01 & 0,06 & 0,03 & $-0,02$ & 0,00 & $-0,10$ & $-0,05$ & 0,06 & 0,08 & $-0,07$ \\
\hline & CieHS & 0,15 & 0,12 & 0,15 & 0,01 & $0,22^{* *}$ & 0,09 & $0,20^{*}$ & 0,16 & 0,15 & $0,20^{*}$ \\
\hline & Ent & $-0,08$ & $-0,12$ & $-0,06$ & $-0,05$ & 0,09 & $-0,11$ & $-0,02$ & $-0,02$ & 0,02 & 0,00 \\
\hline
\end{tabular}

Legenda: CieEx: Ciências Exatas; ArtCom: Artes e Comunicação; CiBioSau: Ciências Biológicas e da Saúde; CiAgrAmb: Ciências Agrárias e Ambientais; Buroc: Atividades Burocráticas; CiHumSoc: Ciências Humanas e Sociais Aplicadas; Entret: Entretenimento.

${ }^{*}=p<0,0,1{ }^{* *}=p<0,05$

No que se refere às diferenças de média entre os gêneros, na amostra completa percebeu-se que houve algumas diferenças significativas, sendo que tais resultados foram obtidos por meio de teste $t$ de Student. No EAP, homens e mulheres se diferenciaram nas dimensões Ciências Exatas $(t=5,709 ; p<0,001)$, Atividades Burocráticas ( $t=3,029, p=0,003)$, Ciências Biológicas e da Saúde $(t=-4,379 ; p<0,001)$ e Ciências Humanas e Sociais Aplicadas ( $t=-2,723 ; p=0,007$ ), sendo que nas duas primeiras dimensões, as maiores médias foram obtidas pelos homens, e nas últimas, pelas mulheres.

Com relação às notas, as disciplinas de Física ( $t=-1,982 ; p=0,049)$, Biologia ( $t=$ $-4,063 ; p=0,000)$, Literatura ( $t=-3,466 ; p=0,001)$, História $(t=-3,944 ; p=0,000)$, Geografia ( $t=-2,924 ; p=0,004)$, Inglês $(t=-3,648 ; p=0,000)$ e Redação $(t=-2,880$; $p=0,004$ ) apresentaram médias com diferenças significativas, sendo que em todas as maiores médias foram obtidas pelas mulheres. 
Entre os estudantes da primeira série do ensino médio, apenas duas correlações significativas e negativas foram encontradas, que podem ser visualizadas na Tabela 3. As notas em História $(r=-0,37, p<0,01)$ e Redação $(r=-0,32 ; p<0,05)$ correlacionaram-se negativamente com a dimensão Ciências Exatas. Levando em consideração apenas os homens da primeira série, duas correlações negativas também foram encontradas, dessa feita entre a dimensão Artes e Comunicação com notas de Matemática $(r=-0,50 ; p<0,05)$ e Português $(r=-0,42 ; p<0,05)$. Já com relação às mulheres $d a$ mesma série, nenhuma correlação significativa foi encontrada.

Tabela 3. Correlações entre notas e dimensões da EAP da primeira série

\begin{tabular}{|c|c|c|c|c|c|c|c|c|c|c|c|}
\hline Gênero & EAP & Matemática & Química & Física & Biologia & Português & Literatura & História & Geografia & Inglês & Redação \\
\hline \multirow[t]{7}{*}{ Geral } & CiEx & $-0,12$ & 0,12 & $-0,14$ & $-0,16$ & $-0,13$ & $-0,06$ & $-0,37^{* *}$ & $-0,04$ & $-0,07$ & $-0,32^{*}$ \\
\hline & ArtCom & $-0,26$ & $-0,17$ & $-0,14$ & $-0,18$ & $-0,19$ & $-0,18$ & $-0,11$ & $-0,16$ & $-0,19$ & $-0,12$ \\
\hline & CiBS & $-0,11$ & $-0,15$ & $-0,08$ & $-0,04$ & $-0,16$ & $-0,09$ & 0,12 & $-0,17$ & $-0,09$ & 0,12 \\
\hline & CiAgAm & $-0,22$ & $-0,11$ & $-0,21$ & $-0,18$ & $-0,19$ & $-0,17$ & $-0,11$ & $-0,20$ & $-0,14$ & $-0,09$ \\
\hline & Bur & $-0,04$ & 0,18 & $-0,05$ & 0,01 & $-0,03$ & 0,09 & $-0,23$ & 0,13 & 0,08 & $-0,23$ \\
\hline & CieHS & $-0,07$ & $-0,05$ & $-0,12$ & 0,06 & 0,04 & $-0,03$ & 0,17 & 0,00 & 0,02 & 0,04 \\
\hline & Ent & $-0,25$ & $-0,17$ & $-0,12$ & $-0,19$ & $-0,20$ & $-0,20$ & $-0,16$ & $-0,11$ & $-0,20$ & $-0,15$ \\
\hline \multirow[t]{7}{*}{ Homens } & CiExat & $-0,20$ & 0,10 & $-0,02$ & $-0,15$ & $-0,23$ & 0,01 & $-0,10$ & $-0,09$ & $-0,08$ & $-0,23$ \\
\hline & ArtCom & $-0,50^{*}$ & $-0,28$ & $-0,28$ & $-0,35$ & $-0,42^{*}$ & $-0,23$ & $-0,11$ & $-0,35$ & $-0,38$ & $-0,25$ \\
\hline & CiBioSau & 0,17 & 0,05 & 0,13 & 0,05 & $-0,07$ & 0,14 & 0,09 & 0,06 & 0,09 & 0,00 \\
\hline & CiAgrAmb & $-0,15$ & $-0,12$ & $-0,05$ & $-0,19$ & $-0,03$ & $-0,10$ & $-0,10$ & $-0,17$ & $-0,07$ & $-0,09$ \\
\hline & Buroc & $-0,17$ & 0,18 & 0,05 & $-0,07$ & $-0,11$ & 0,09 & 0,05 & 0,06 & 0,04 & $-0,07$ \\
\hline & CieHumSoc & $-0,20$ & $-0,14$ & $-0,13$ & $-0,14$ & $-0,12$ & $-0,16$ & 0,10 & $-0,23$ & $-0,15$ & $-0,11$ \\
\hline & Entret & $-0,40$ & $-0,20$ & $-0,13$ & $-0,36$ & $-0,33$ & $-0,28$ & $-0,09$ & $-0,28$ & $-0,29$ & $-0,16$ \\
\hline \multirow[t]{7}{*}{ Mulheres } & CiExat & $-0,10$ & 0,06 & $-0,26$ & $-0,04$ & $-0,12$ & $-0,03$ & $-0,34$ & $-0,00$ & 0,03 & $-0,27$ \\
\hline & ArtCom & $-0,16$ & $-0,10$ & $-0,08$ & $-0,19$ & $-0,09$ & $-0,23$ & $-0,28$ & $-0,10$ & $-0,15$ & $-0,17$ \\
\hline & CiBioSau & $-0,26$ & $-0,17$ & $-0,22$ & $-0,21$ & $-0,25$ & $-0,34$ & $-0,05$ & $-0,33$ & $-0,27$ & 0,04 \\
\hline & CiAgrAmb & $-0,27$ & $-0,03$ & $-0,35$ & $-0,26$ & $-0,29$ & $-0,29$ & $-0,28$ & $-0,22$ & $-0,24$ & $-0,21$ \\
\hline & Buroc & 0,03 & 0,15 & $-0,13$ & 0,10 & 0,02 & 0,10 & $-0,34$ & 0,17 & 0,14 & $-0,32$ \\
\hline & CieHumSoc & $-0,01$ & 0,05 & $-0,14$ & 0,09 & 0,14 & $-0,01$ & 0,03 & 0,13 & 0,06 & $-0,04$ \\
\hline & Entret & $-0,18$ & $-0,14$ & $-0,13$ & $-0,15$ & $-0,15$ & $-0,18$ & $-0,30$ & $-0,02$ & $-0,18$ & $-0,23$ \\
\hline
\end{tabular}

Legenda: CieEx: Ciências Exatas; ArtCom: Artes e Comunicação; CiBioSau: Ciências Biológicas e da Saúde; CiAgrAmb: Ciências Agrárias e Ambientais; Buroc: Atividades Burocráticas; CiHumSoc: Ciências Humanas e Sociais Aplicadas; Entret: Entretenimento.

${ }^{*}=p<0,0,1 \quad{ }^{* *}=p<0,05$

Com relação à amostra do primeiro ano, as dimensões do EAP Ciências Exatas ( $t=$ 5,386; $p<0,001)$, Artes e Comunicação ( $t=-2,106 ; p=0,040)$, Ciências Biológicas e da Saúde ( $t=-3,095 ; p=0,003)$, e Ciências Humanas e Sociais Aplicadas $(t=-3,835 ; p<0,000)$ apresentaram diferença significativa de médias, sendo que apenas na primeira dimensão os homens obtiveram maiores médias. Já no tocante às notas da mesma 
série, apenas História $(t=-2,478 ; p=0,016)$ e Redação $(t=-0,261 ; p=0,028)$ apresentaram médias significativamente diferentes, sempre com vantagem para as mulheres.

Nos dados da segunda série, apresentados na Tabela 4, novamente Redação se correlacionou negativamente com Ciências Exatas ( $r=-0,21 ; p<0,05)$. Outras correlações significativas foram encontradas entre a dimensão Ciências Humanas e Sociais e Física $(r=0,26 ; p<0,01)$ e História $(r=0,31 ; p<0,01)$. Tomando os escores masculinos dessa série, pode-se observar que Ciências Exatas se correlacionou negativamente com Português ( $r=-0,49 ; p<0,05)$, Geografia $(r=-0,39 ; p<0,05)$ e Redação $(r=-0,47 ; 0<0,05)$. Outras correlações foram encontradas, a saber, Ciências Agrárias e Ambientais com Redação $(r=0,53 ; p<0,05)$ e Entretenimento com Inglês $(r=-0,43 ; p<0,05)$. Com relação às mulheres, foram observadas correlações entre Ciências Biológicas e da Saúde e Química $(r=0,27$; $p<0,05)$, Ciências Agrárias e Ambientais com Matemática $(r=0,26 ; p<0,05)$, e Ciências Humanas e Sociais Aplicadas com Física $(r=0,31 ; p<0,01)$ e História $(r=0,32 ; p<0,01)$.

Tabela 4. Correlações entre notas e dimensões da EAP da segunda série

\begin{tabular}{|c|c|c|c|c|c|c|c|c|c|c|c|}
\hline Gênero & EAP & Matemática & Química & Fisica & Biologia & Português & Literatura & História & Geografia & Inglês & Redação \\
\hline \multirow[t]{7}{*}{ Geral } & CiEx & $-0,02$ & $-0,02$ & $-0,15$ & $-0,20$ & $-0,09$ & $-0,04$ & $-0,19$ & $-0,09$ & $-0,03$ & $-0,21^{*}$ \\
\hline & ArtCom & 0,06 & 0,02 & 0,09 & 0,00 & 0,12 & 0,06 & 0,15 & 0,02 & 0,05 & $-0,07$ \\
\hline & CiBS & 0,13 & 0,17 & 0,11 & 0,12 & 0,04 & 0,11 & 0,05 & 0,08 & 0,09 & 0,00 \\
\hline & CiAgAm & 0,12 & 0,06 & 0,01 & $-0,03$ & 0,06 & 0,03 & $-0,07$ & $-0,02$ & $-0,06$ & $-0,03$ \\
\hline & Bur & $-0,12$ & $-0,06$ & $-0,05$ & $-0,13$ & $-0,11$ & $-0,12$ & $-0,12$ & $-0,12$ & $-0,03$ & $-0,16$ \\
\hline & CieHS & 0,12 & 0,12 & $0,26^{* *}$ & 0,09 & 0,13 & 0,10 & $0,31^{* *}$ & 0,15 & 0,14 & 0,19 \\
\hline & Ent & $-0,01$ & $-0,10$ & $-0,02$ & $-0,04$ & 0,04 & $-0,04$ & 0,03 & $-0,06$ & $-0,03$ & $-0,15$ \\
\hline \multirow[t]{7}{*}{ Homens } & CiExat & $-0,06$ & $-0,19$ & $-0,33$ & $-0,35$ & $-0,49^{*}$ & 0,02 & $-0,39$ & $-0,39^{*}$ & $-0,06$ & $-0,47^{*}$ \\
\hline & ArtCom & 0,01 & $-0,14$ & 0,01 & 0,01 & $-0,06$ & $-0,24$ & 0,19 & $-0,04$ & $-0,11$ & $-0,15$ \\
\hline & CiBioSau & $-0,13$ & $-0,24$ & $-0,09$ & $-0,27$ & $-0,34$ & $-0,09$ & $-0,30$ & $-0,36$ & $-0,13$ & $-0,36$ \\
\hline & CiAgrAmb & $-0,19$ & $-0,34$ & $-0,21$ & $-0,39$ & $-0,39$ & $-0,22$ & $-0,39$ & $-0,38$ & $-0,32$ & $-0,53^{*}$ \\
\hline & Buroc & $-0,22$ & $-0,07$ & $-0,11$ & $-0,22$ & $-0,38$ & 0,11 & $-0,21$ & $-0,37$ & $-0,04$ & $-0,05$ \\
\hline & CieHumSoc & $-0,23$ & 0,00 & 0,09 & $-0,05$ & $-0,10$ & $-0,16$ & 0,26 & 0,02 & $-0,09$ & 0,19 \\
\hline & Entret & $-0,21$ & $-0,31$ & $-0,34$ & $-0,25$ & $-0,28$ & $-0,22$ & $-0,22$ & $-0,30$ & $-0,43^{*}$ & $-0,25$ \\
\hline \multirow[t]{7}{*}{ Mulheres } & CiExat & 0,03 & 0,08 & $-0,01$ & $-0,05$ & 0,03 & 0,01 & $-0,05$ & 0,09 & 0,05 & $-0,04$ \\
\hline & ArtCom & 0,06 & 0,06 & 0,11 & $-0,01$ & 0,17 & 0,14 & 0,14 & 0,03 & 0,08 & $-0,03$ \\
\hline & CiBioSau & 0,17 & $0,27^{*}$ & 0,11 & 0,18 & 0,18 & 0,10 & 0,09 & 0,20 & 0,09 & 0,25 \\
\hline & CiAgrAmb & $0,26^{*}$ & 0,23 & 0,14 & 0,17 & 0,22 & 0,15 & 0,08 & 0,16 & 0,07 & 0,16 \\
\hline & Buroc & $-0,05$ & $-0,01$ & 0,04 & $-0,03$ & $-0,06$ & $-0,12$ & $-0,02$ & 0,03 & 0,04 & $-0,11$ \\
\hline & CieHumSoc & 0,22 & 0,14 & $0,31^{* *}$ & 0,11 & 0,21 & 0,16 & $0,32^{* *}$ & 0,20 & 0,20 & 0,18 \\
\hline & Entret & 0,05 & $-0,04$ & 0,09 & 0,02 & 0,14 & 0,01 & 0,12 & 0,03 & 0,10 & $-0,09$ \\
\hline
\end{tabular}

Legenda: CieEx: Ciências Exatas; ArtCom: Artes e Comunicação; CiBioSau: Ciências Biológicas e da Saúde; CiAgrAmb: Ciências Agrárias e Ambientais; Buroc: Atividades Burocráticas; CiHumSoc: Ciências Humanas e Sociais Aplicadas; Entret: Entretenimento. ${ }^{*}=p<0,0,1 \quad{ }^{* *}=p<0,05$ 
Os alunos da segunda série obtiveram médias diferenciadas, segundo o teste $t$, nas dimensões Ciências Exatas ( $t=2,223 ; p=0,029)$, Atividades Burocráticas $(t=2,356 ; p=$ $0,021)$ e Ciências Biológicas e da Saúde $(t=-2,526 ; p=0,013)$, sendo que apenas na última as maiores médias foram obtidas pelas mulheres. Observando-se as médias das notas dos alunos dessa série, percebem-se diferenças em Física $(t=-2,239 ; p=0,027)$, Biologia $(t=-3,108 ; p=0,002)$, Literatura $(t=-2,348 ; p=0,021)$, História $(t=-2,188 ; p=$ $0,031)$, Geografia $(t=-1,957 ; p=0,053)$ e Inglês $(t=-2,587 ; p=0,011)$, havendo mais uma vez predominância das mulheres com médias mais altas em relação aos homens.

Já no terceiro ano (Tabela 5), um maior número de correlações significativas foi encontrado, envolvendo cinco das sete dimensões do EAP e seis das 10 disciplinas avaliadas. A dimensão Ciências Biológicas e da Saúde mostrou-se correlacionada positivamente com as notas de Biologia $(r=0,22 ; p<0,05)$ e Geografia $(r=0,24$; $p<0,05)$, que também se correlacionou com Ciências Agrárias e Ambientais ( $r=0,24$; $p<0,05)$, enquanto que a dimensão Burocráticas correlacionou-se com Física $(r=0,24$; $p<0,05)$. A dimensão Ciências Humanas e Sociais correlacionou-se positivamente com Português $(r=0,25 ; p<0,05)$, Geografia $(r=0,24 ; p<0,05)$ e Redação $(r=0,26$; $p<0,05)$. Na dimensão Entretenimento observou-se a única correlação negativa, que foi com História $(r=0,22 ; p<0,05)$.

Considerando apenas os estudantes do sexo masculino dessa série, Atividades Burocráticas se correlacionou positivamente com Física $(r=0,38 ; p<0,05)$, Português $(r=0,33 ; p<0,05)$ e Redação $(r=0,33 ; p<0,05)$, enquanto que Entretenimento mostrou-se negativamente correlacionada com as notas de História ( $r=-0,35$; $p<0,05)$. Já entre mulheres da terceria série, percebe-se que os interesses por Ciências Exatas se correlacionaram com Química $(r=0,30 ; p<0,05)$, Física $(r=0,32$; $p<0,05)$, Biologia $(r=0,36 ; p<0,05)$ e História $(r=0,31 ; p<0,05)$; Ciências Biológicas e da Saúde, com Biologia $(r=0,38 ; p<0,05)$ e Geografia $(r=0,33 ; p<0,05)$; Ciências Agrárias e Ambientais com Biologia ( $r=0,39 ; p<0,05)$, Português $(r=0,42 ; p<0,05)$, Geografia $(r=0,44 ; p<0,05)$ e Redação $(r=0,42 ; p<0,05)$; por fim, Ciências Humanas e Sociais Aplicadas se correlacionou com Matemática $(r=0,32 ; p<0,05)$, Português $(r=0,37 ; p<0,05)$, História $(r=0,31 ; p<0,05)$ e Redação $(r=0,37 ; p<0,05)$.

Tabela 5. Correlações entre notas e dimensões da EAP da terceira série

\begin{tabular}{cccccccccccc}
\hline Cênero & EAP & Matemática & Química & Física & Biologia & Português & Literatura & História & Ceografia & Inglês & Redação \\
\hline Geral & CiEx & 0,15 & 0,12 & 0,18 & 0,21 & 0,11 & 0,09 & 0,09 & $-0,04$ & $-0,01$ & 0,11 \\
& ArtCom & 0,03 & $-0,09$ & $-0,05$ & $-0,18$ & 0,21 & 0,00 & 0,02 & 0,03 & 0,04 & 0,21 \\
& CiBS & 0,16 & 0,13 & 0,00 & $0,22^{*}$ & 0,02 & 0,19 & $-0,04$ & $0,24^{*}$ & $-0,04$ & 0,03 \\
& CiAgAm & 0,17 & 0,14 & 0,03 & 0,18 & 0,18 & 0,18 & 0,07 & $0,24^{*}$ & $-0,10$ & 0,18 \\
& Bur & 0,14 & 0,10 & $0,24^{*}$ & $-0,00$ & 0,14 & $-0,01$ & 0,02 & 0,009 & 0,10 & 0,13 \\
& CieHS & 0,22 & 0,11 & 0,13 & $-0,03$ & $0,25^{*}$ & 0,19 & 0,15 & $0,24^{*}$ & 0,09 & $0,26^{*}$ \\
& Ent & $-0,06$ & $-0,10$ & $-0,08$ & $-0,19$ & 0,15 & $-0,14$ & $-0,22^{*}$ & $-0,08$ & $-0,03$ & 0,16 \\
\hline
\end{tabular}


Tabela 5. (continuação)

\begin{tabular}{|c|c|c|c|c|c|c|c|c|c|c|c|}
\hline \multirow[t]{7}{*}{ Homens } & CiExat & 0,17 & 0,07 & 0,16 & 0,14 & 0,23 & 0,11 & $-0,01$ & 0,03 & 0,04 & 0,23 \\
\hline & ArtCom & $-0,06$ & $-0,14$ & $-0,16$ & $-0,19$ & 0,12 & 0,06 & $-0,19$ & $-0,01$ & $-0,13$ & 0,13 \\
\hline & $\begin{array}{c}\text { CiBio- } \\
\text { Sau }\end{array}$ & $-0,01$ & $-0,03$ & $-0,22$ & $-0,04$ & $-0,13$ & $-0,02$ & $-0,28$ & $-0,07$ & $-0,20$ & $-0,12$ \\
\hline & $\begin{array}{l}\text { CiA- } \\
\text { grAmb }\end{array}$ & 0,09 & 0,05 & $-0,09$ & 0,01 & 0,07 & 0,11 & $-0,08$ & 0,13 & $-0,16$ & 0,08 \\
\hline & Buroc & 0,23 & 0,13 & $0,38^{*}$ & 0,07 & $0,33^{*}$ & 0,31 & $-0,05$ & 0,11 & 0,19 & $0,33^{*}$ \\
\hline & $\begin{array}{c}\text { Cie- } \\
\text { HumSoc }\end{array}$ & 0,11 & 0,01 & 0,09 & $-0,04$ & 0,10 & 0,25 & 0,01 & 0,20 & $-0,07$ & 0,11 \\
\hline & Entret & $-0,12$ & $-0,10$ & $-0,06$ & $-0,21$ & 0,03 & $-0,02$ & $-0,35^{*}$ & $-0,09$ & $-0,18$ & 0,04 \\
\hline \multirow[t]{7}{*}{ Mulheres } & CiExat & 0,19 & $0,30^{*}$ & $0,32^{*}$ & $0,36^{*}$ & 0,27 & 0,20 & $0,31^{*}$ & 0,11 & 0,20 & 0,27 \\
\hline & ArtCom & 0,09 & $-0,08$ & 0,04 & $-0,19$ & 0,25 & $-0,09$ & 0,19 & 0,01 & 0,15 & 0,25 \\
\hline & $\begin{array}{c}\text { CiBio- } \\
\text { Sau }\end{array}$ & 0,24 & 0,19 & 0,16 & $0,38^{*}$ & 0,00 & 0,25 & 0,09 & $0,33^{*}$ & $-0,06$ & 0,00 \\
\hline & $\begin{array}{l}\text { CiA- } \\
\text { grAmb }\end{array}$ & 0,27 & 0,28 & 0,20 & $0,39^{*}$ & $0,42^{* *}$ & $0,31^{*}$ & 0,30 & $0,44^{* *}$ & 0,02 & $0,42^{* *}$ \\
\hline & Buroc & 0,10 & 0,13 & 0,16 & $-0,04$ & 0,11 & $-0,17$ & 0,14 & 0,04 & 0,18 & 0,11 \\
\hline & $\begin{array}{c}\text { Cie- } \\
\text { HumSoc }\end{array}$ & $0,32^{*}$ & 0,20 & 0,16 & $-0,04$ & $0,37^{*}$ & 0,09 & $0,31^{*}$ & 0,23 & 0,19 & $0,37^{*}$ \\
\hline & Entret & $-0,03$ & $-0,13$ & $-0,13$ & $-0,19$ & 0,26 & $-0,29$ & $-0,11$ & $-0,11$ & 0,07 & 0,26 \\
\hline
\end{tabular}

Legenda: CieEx: Ciências Exatas; ArtCom: Artes e Comunicação; CiBioSau: Ciências Biológicas e da Saúde; CiAgrAmb: Ciências Agrárias e Ambientais; Buroc: Atividades Burocráticas; CiHumSoc: Ciências Humanas e Sociais Aplicadas; Entret: Entretenimento. ${ }^{*}=p<0,0,1 \quad{ }^{* *}=p<0,05$

No terceiro ano, com relação às dimensões do EAP, foram percebidas diferenças significativas apenas em Ciências Exatas ( $t=2,761 ; p=0,007$ ), e novamente com vantagem dos homens. Quanto às notas, Português ( $t=-3,881 ; p=0,000)$, Literatura $(t=-2,106 ; p=0,038)$, Geografia $(t=-3,176 ; p=0,002)$, Inglês $(t=-3,569 ; p=0,001)$ e Redação ( $t=-3,930 ; p=0,000)$ se mostraram diferenciadas, sempre com médias mais altas das mulheres.

\section{Discussão}

Pode-se perceber que, de forma geral, as correlações parecem coerentes, embora baixas, uma vez que evidenciam correlações negativas entre áreas com poucas relações de conteúdo, e coeficientes positivos com disciplinas e dimensões afins. Assim como no estudo de Noronha, Sisto e Santos (2007), que encontraram coerências entre a área de estudos de universitários e seus interesses, o presente estudo também parece ir nesse sentido. Apenas a correlação significativa entre Ciências Humanas e Sociais Aplicadas e Física, encontrada na amostra geral das três séries, parece destoar desse contexto, devendo ser atribuido a fatores intrínsecos da amostra. 
Com relação às diferenças entre gêneros, os resultados encontrados nessa pesquisa estão de acordo com os achados de Welter (2007), Fogliatto e colaboradores (2003), Styer (1995) e Gottfredson (1981), que embora tenham avaliado amostras distintas do presente estudo, afirmaram a existência de diferenças substanciais nos interesses entre os gêneros, sugerindo uma preferência de homens por atividades mais lógicas, exatas, administrativas e tecnológicas, enquanto que mulheres parecem preferir atividades mais sociais, intelectuais, com pouco esforço físico e sem demanda de objetividade e rotina. Entretanto, vale ressaltar que Sartori (2007), utilizando o EAP em seu trabalho, encontrou resultados destoantes, uma vez que não foram observadas diferenças significativas de gênero. Talvez, o fato de a amostra do presente estudo ter sido maior pode ter favorecido o aparecimento de tais diferenças estatisticamente significativas.

No tocante às notas, percebeu-se que as mulheres mostraram melhor desempenho acadêmico, sendo que Dias e colaboradores (2006) e Plazas e colaboradores (2006) já haviam encontrado resultados semelhantes. É interessante notar que, embora os homens tenham maior preferência por atividades exatas, as notas de física das mulheres da amostra geral e da segunda série do presente estudo foram melhores do que as dos homens, bem como o desempenho em aritmética delas.

Ainda, pode-se perceber que a quantidade de correlações significativas tende a aumentar, quanto maior o nível de escolarização. Com relação ao primeiro ano, na amostra total encontrou-se apenas duas correlações significativas, contra três da segunda série e oito da terceira. Nas amostras masculinas, no primeiro ano observaram-se duas correlações, cinco no segundo e quatro no terceiro, enquanto que nas femininas, nenhuma correlação foi encontrada no primeiro ano, quatro no segundo e 15 no terceiro. Se uma nota escolar for tomada como medida de habilidade em determinada disciplina, pode-se pensar que a correlação entre ela e os interesses informa sobre o ajustamento ou não do sujeito a determinadas atividades, além de mostrar em alguma medida que os sujeitos têm um autoconhecimento apropriado, uma vez que preferem atividades que tendem a desempenhar melhor. Assim, os resultados apresentados parecem estar de acordo com os achados de Neiva e colaboradores (2005), uma vez que parecem indicar que, quanto mais escolarizados, mais os interesses parecem se cristalizar e se tornar mais coerentes com as próprias habilidades, que são pressupostos do desenvolvimento vocacional. Considerando que o objetivo dessa pesquisa não foi avaliar a maturidade vocacional, parece pertinente que outras análises sejam realizadas, com a inclusão de instrumentos de coleta de dados apropriados para tal fim.

Em síntese, sobre as relações entre as variáveis de desempenho escolar e interesses profissionais, parece haver relações entre elas, ao menos na amostra estudada. 
Enquanto outros estudos já haviam encontrado relações entre desempenho e autoconceito (Silva \& Vendramini, 2005), compreensão de leitura (Oliveira \& Santos, 2006), status sociométrico (Plazas \& cols. (2006) e depressão (Pérez \& Urquijo, 2001), o presente estudo encontrou correlações significativas entre disciplinas e dimensões de interesse que parecem ter relação entre si.

\section{Considerações finais}

A OP, inserida no contexto escolar-educacional, cada vez mais assume uma importância no sentido de favorecer aos jovens a possibilidade de escolha ajustada e adequada às suas condições, características e necessidades. Dessa forma, é necessário que se pense em programas de OP dentro do currículo educacional, tanto de escolas públicas como de particulares, visando integrar ações educativas, avaliativas e interventivas dentro do contexto social e escolar em que o aluno está inserido.

Nesse sentido, ao avaliar os interesses em relação ao desempenho escolar o presente artigo visa contribuir para que as ações práticas nesse âmbito possam ter um caráter mais integrativo, utilizando procedimentos de avaliação escolar para ajudar a compreender a relação do sujeito com as diferentes disciplinas e suas especialidades. Além disso, ao avaliar as diferenças entre os gêneros, os resultados do presente estudo indicam a possiblidade de se pensar em intervenções específicas para grupos de homens ou mulheres.

Para futuras investigações, além da sugestão indicada anteriormente, propõe-se que estudos longitudinais sejam feitos no mesmo sentido, para que se possa avaliar a estabilidade dos interesses ao longo do processo de escolarização secundária.

\section{Referências Bibliográficas}

Abade, F. L. (2005). Orientação Profissional no Brasil: Uma Revisão Histórica da Produção Científica. Revista Brasileira de Orientação Profissional, 6(1), 15-24.

Anastasi, A., \& Urbina, S. (2000). Testagem Psicológica. Porto Alegre: Artes Médicas.

Conselho Federal de Psicologia - CFP (2001). Resolução n. ${ }^{\circ}$ 25/2001. Disponível em www.pol. org.br. Acessado em 17 de março de 2008.

Conselho Federal de Psicologia - CFP (2003). Resolução n. ${ }^{\circ}$ 02/2003. Disponível em www. pol.org.br. Acessado em 17 de março de 2008.

Conselho Federal de Psicologia - CFP (2008). Sistema de Avaliação de Testes Psicológicos. Disponivel em www.pol.org.br. Acessado em 31 de março de 2008. 
Dias, T. L., Enumo, S. R. F., \& Turini, F. A. (2006). Avaliação do desempenho acadêmico de alunos do ensino fundamental em Vitória, Espírito Santo. Estudos de psicologia (Campinas), 23(4), 381-390.

Fogliatto, H., Pérez, E., Olaz, F., \& Parodi, L. (2003). Cuestionario de Intereses Profesionales Revisado (CIP-R). Análisis de sus Propiedades Psicométricas. Evaluar, 3, 61-79.

Freitas, E. (1973). Origens e organização do ISOP. Arquivos Brasileiros de Psicologia Aplicada, 25(1), 7-16.

Gottfedson, L. (1981) Circumscription and compromise: a developmental theory of occupational aspirations. Journal of Counseling Psychology, 28 (6), 545-479.

Holland, J. L. (1963). Explorations of a theory of vocational choice and achievement: II. A four-year prediction study. Psychological Reports, 12, 547-594.

Holland, J. L.; Powell, A. B., \& Fritzsche, B. A. (1994). SDS Self-Directed Search Professional user's guide. Florida: Psychological Assessment Resources, Inc.

Melo-Silva, L. L (1999). Pesquisas brasileiras no domínio da orientação vocacional e profissional. Disponivel em http://www.aiospconference2005.pt/abstracts/178.pdf. Consulta feita em março/2006.

Melo-Silva, L. L.; Bonfim, T. A., Esbrogeo, M. C., \& Soares, D. H. P. (2003). Um estudo preliminar sobre práticas em orientação profissional. Revista Brasileira de Orientação Profissional, 4(1-2), 21-34.

Neiva, K. M. C., Silva, M. B., Miranda, V. R., \& Esteves, C. (2005). Um estudo sobre a maturidade para a escolha profissional de alunos do ensino médio. Revista Brasileira de Orientação Profissional, 6(1), 1-14.

Noronha, A. P. P. \& Ambiel, R. A. M. (2006). Orientação profissional e vocacional: análise da produção científica. PsicoUsf, 11 (1) 75-84.

Noronha, A. P. P., Sisto F. F., \& Santos, A. A. A. (2007). Escala de Aconselhamento Profissional (EAP): manual técnico. São Paulo: Vetor Editora.

Ottati, F., Noronha, A. P. P., \& Salviati, M. (2003). Testes psicológicos: qualidade de instrumentos de interesse profissional. Interação, 7 (1), 89-96.

Pérez, M. V., \& Urquijo, S. (2001) Depresión en adolescentes: relaciones con el desempeño acadêmico. Psicologia escolar e educacional, 5(1), 49-58.

Plazas, E. A., Penso, R. A., \& López, S. E. (2006). Relación entre estatus sociométrico, género y rendimiento acadêmico. Psicología desde el caribe, 17, 176-195.

Santos, P. J. (1997). Adolescência e indecisão vocacional. Dissertação de Mestrado, Universidade do Porto, Porto.

Sartori, F. A. (2007). Estudo correlacional entre a Escala de Aconselhamento Profissional (EAP) e o SDS. Dissertação de mestrado, Universidade São Francisco, Itatiba.

Savickas, M. L. (1999). The psychology of interests. In M. L. Savickas \& A. R. Spokane (eds.), Vocational interests. Meanings, Measurement and Counseling Use (pp. 19-56). Palo Alto, CA: Davies-Black.

Welter, G. M. (2007). O BBT: Teste de Fotos de Profissões em Adultos e Adolescentes. Revista Brasileira de Orientação Profissional, 8(1), 45-58. 


\section{Relations between high school students' scholastic performance and professional interests \\ Scholastic performance and professional interests}

This article searched to study the relations between professional interests and grades of students from a private high school of Minas Gerais state, and the means differences between gender. Therefore, 245 students (24,1\% from first class, 40,8\% from second, e 35,1\% from third) were subjects in the research, with mean age of 16,3 years $(S D=0,9)$, and $60,8 \%$ were women. The instrument used was Escala de Aconselhamento Profissional (EAP), and the grades corresponding to the two initial bimesters of 2005. Results showed coherent significant correlations, with positive correlation between relatives courses and interests areas, such as negatives ones between areas with low degree of relative contents. A tendency to prefer typical gender activities was fulfilled in this sample, as well a better scholastic performance by women was observed. Practical implications and new studies in the direction to clarify such relations are indicated.

KEYWORDS: High School; Gender Differences; Psychological Assessment; Vocational Counseling. 